\title{
Author Corrections: Reconstruction of cell spatial organization from single-cell RNA sequencing data based on ligand-receptor mediated self-assembly
}

Xianwen Ren, Guojie Zhong, Qiming Zhang, Lei Zhang, Yujie Sun and Zemin Zhang (iD

(c) CEMCS, CAS 2021

Cell Research (2021) 31:1319-1320; https://doi.org/10.1038/s41422-021-00550-5

Correction to: Cell Research https://doi.org/10.1038/s41422-0200353-2, published online 15 June 2020

We apologize for an error that we just found in the paper published online on 15 June 2020. The mouse ligand receptor database we used contained duplicated pairs, this will affect the Fig. 2b, Supplementary information, Figs. S1b, S1c, S2 and S3. We updated the mouse ligand-receptor database and the correct version is provided below.

The update on Fig. $2 \mathrm{~b}$ does not affect the conclusion of that part.

The "Quantitative evaluation based on the scRNA-seq data of mouse liver lobules showed that CSOmap reached high a human pancrea

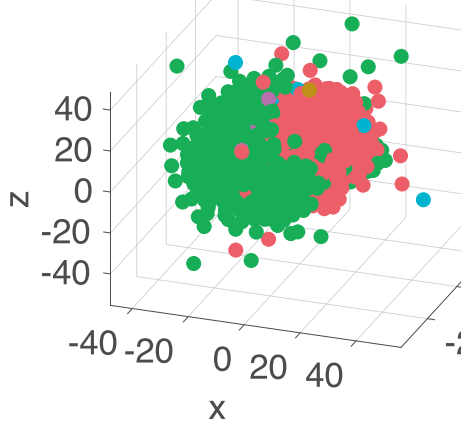

b Mouse Pancrea

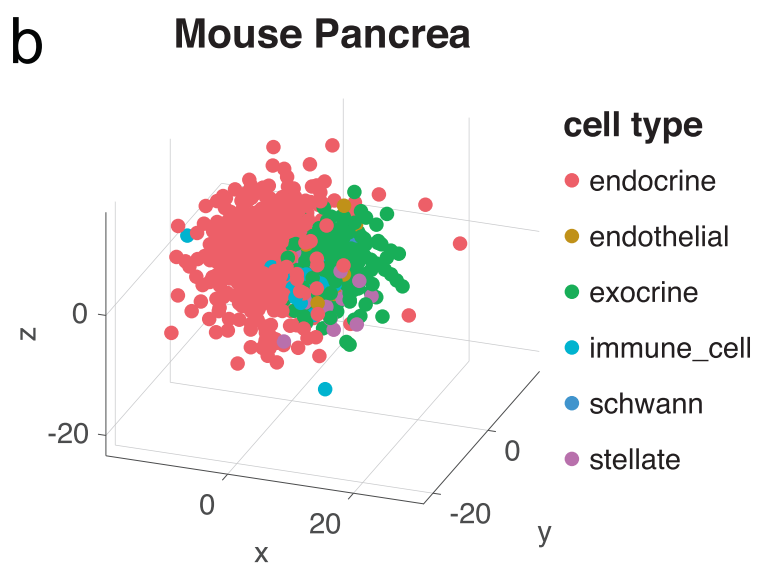

\section{cell type}

- endocrine

- endothelial

- exocrine

- immune_cell

20 schwann

y
- stellate
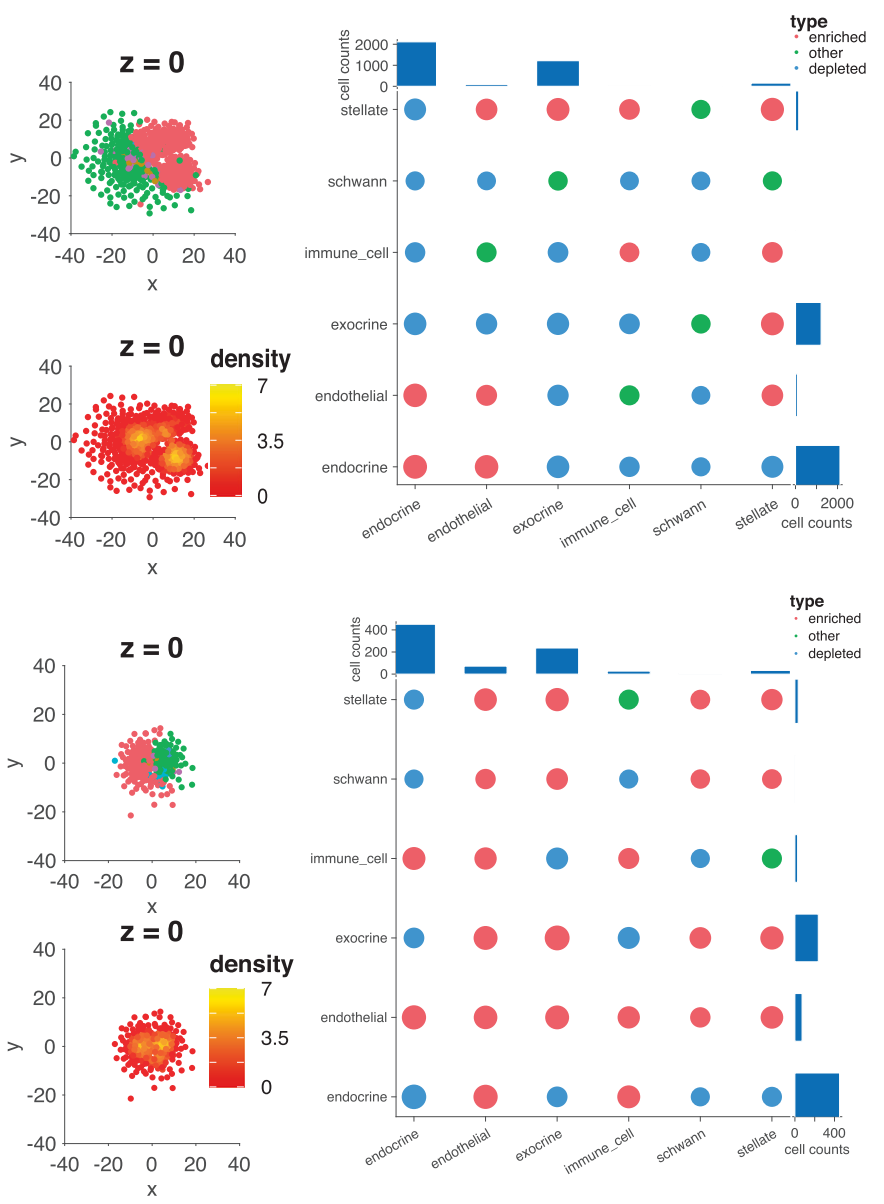
1320

consistence $(R=0.85, P<0.01$, Spearman correlation, Supplementary information, Fig. S1b) with the reference." part in the article should be updated to " $R=0.96, P<0.01$, Pearson correlation, Supplementary information, Fig. S1b)".

The "In silico predictions by CSOmap reached consistent results with paired-cell sequencing $(R=0.73, P<0.04$, Spearman correlation, Supplementary information, Fig. S1c)" part should be updated to " $R=0.75, P<0.04$, Pearson correlation, Supplementary information, Fig. S1c)".

The "Systematic evaluation based on the Tabula Muris datasets demonstrated that CSOmap could reproduce the organ-level separations by revealing significantly higher intra-organ cellular interactions than inter-organ interactions for almost all organs except tongue $(15 / 16,93.75 \%$, Supplementary information, Fig. S2), of which 176 out of 199 interacting cell type pairs were from different cell types." should be updated to "for almost all organs except tongue and large intestine (14/16, 87.5\%, Supplementary information, Fig. S2), of which 225 out of 262 interacting cell type pairs were from different cell types".

Update on Supplementary information, Fig. S3 does not affect the description of the corresponding part.

Overall, these corrections do not affect the conclusion of this article.

\section{ADDITIONAL INFORMATION}

Supplementary information The online version contains supplementary material available at https://doi.org/10.1038/s41422-021-00550-5. 